\title{
Review: selegiline leads to a small short term improvement in cognition and activities of daily living in Alzheimer's disease
}

\author{
Wilcock GK, Birks J, Whitehead A, et al. The effect of selegiline in the treatment of people with Alzheimer's disease: a \\ meta-analysis of published trials. Int J Geriatr Psychiatry 2002 Feb;17:175-83.
}

QUESTION: In patients with Alzheimer's disease, does selegiline improve cognitive performance, functional ability, emotional state, and global response?

\section{Data sources}

Studies were identified by searching Medline, EMBASE/ Excerpta Medica, PyscLIT, the Cochrane Controlled Trials Register, reference lists of retrieved articles, and conference proceedings; and by contacting pharmaceutical companies and authors.

\section{Study selection}

Studies were selected if they were unconfounded, double blind, randomised controlled trials that compared selegiline with placebo in patients with Alzheimer's disease. Studies had to have been reported before the end of 1998; patients had to meet NINCDSADRDA or DSM criteria for Alzheimer's disease; and any secondary treatments (eg, physostigmine and lecithin) had to be given to both the selegiline and placebo groups.

\section{Data extraction}

Individual patient data were requested. When these data were not provided, summary data were extracted from the published reports for patient characteristics, treatment completion, and outcomes.

\section{Main results}

14 studies (1073 patients) met the selection criteria, and individual patient data were available for 8 studies $(821$ patients). 5 studies used a crossover design. Study duration ranged from 3 weeks to 2 years, and the selegiline dose was $10 \mathrm{mg} /$ day. Patients' mean age ranged from $65-83$ years. Selegiline was better than placebo for $\operatorname{cog}$ nition at 4-6 weeks and 8-17 weeks (table), but the groups did not differ at 21-30 weeks or 76-69 weeks. Selegiline led to a benefit for activities of daily living at 4-6 weeks, but the benefit disappeared at later assessments (table). The groups did not differ for emotional state or global assessment.

\section{Conclusions}

In patients with Alzheimer's disease, selegiline leads to a small short term improvement in cognition and activities of daily living. Selegiline does not improve emotional state or global response.

\section{COMMENTARY}

Wilcock et al did an exemplary job using the tools of metaanalysis to combine data from studies that used varying inclusion criteria and outcome measures. This article and the closely related review in the Cochrane Library ${ }^{1}$ provide strong evidence that, at best, the effect of selegiline in Alzheimer's disease is of little clinical significance. Not only were the magnitudes of the improvements in cognition and activities of daily living seen with selegiline treatment small and of no clinical significance, they were both largely dependent on a single study that was inconsistent with the results of the other included trials. Although few adverse events attributable to selegiline occurred, the lack of clinically significant efficacy offers little hope that further studies are warranted.

This article illustrates the value of using meta-analysis to allow relatively confident conclusions to be drawn from studies that are individually small and that use varying outcome measures. Selegiline treatment was based on sound theoretical grounds, and it is disappointing that it turned out to have little utility.

Jay S Luxenberg, MD Director of Medical Services, Jewish Home San Francisco California USA

1 Birks J, Flicker L. Selegiline for Alzheimer's disease Cochrane Database Syst Rev 2002:(1):CD000442.
Source of funding:

Council, UK

For correspondence: Dr G K Wilcock,

University of Bristol, Bristol, UK. gordon.wilcock@ bris.ac.uk
Selegiline v placebo for Alzheimer's disease

\begin{tabular}{lll} 
Outcomes & Number of studies & Standardised mean difference $(95 \%$ Cl) \\
Cognition at 4-6 weeks & 8 & $0.39(0.07 \text { to } 0.72)^{*}$ \\
\hline Cognition at $8-17$ weeks & 10 & $0.45(0.03$ to 0.88$)$ \\
\hline Activities of daily living at 4-6 weeks & $6^{*}$ & $0.27(0.12 \text { to } 0.40)^{*}$ \\
\hline Activities of daily living at $8-17$ weeks & 7 & $0.33(-0.33$ to 0.69$) \dagger$ \\
\hline
\end{tabular}

*Data provided by author; all standardized mean differences favour selegiline.

†Not statistically significant. 